\title{
PREVALÊNCIA DO ANTI-HBS E AVALIAÇÃO DA RESPOSTA SOROLÓGICA À VACINA CONTRA HEPATITE B EM PACIENTES CANDIDATOS AO TRANSPLANTE HEPÁTICO NO HOSPITAL SANTA ISABEL DE BLUMENAU/SANTA CATARINA
}

\author{
Anti-HBs prevalence and assessment of the response to serological Hepatitis $B$ vaccine in \\ patients eligible for liver transplantation at Hospital Santa Isabel of Blumenau/Santa Catarina
}

\author{
Marcelo Augusto Scheidemantel Nogara, Luiza Dadan Perini, Fabiane Miura Ogg De Salles, Maria Gabriela Ortiz de Noronha, \\ Guilherme Bortolli Seter, Camila Ribeiro Batista
}

\section{RESUMO}

Introdução: Anti-HBs reagente pode ser um fator protetor para pacientes candidatos a transplante hepático porque representa imunidade contra o vírus da hepatite B (VHB). Após as três doses da vacina, mais de $90 \%$ dos adultos jovens desenvolvem respostas adequadas de anticorpos Anti-HBs. Objetivos: Identificar a prevalência daquele anticorpo e avaliar a resposta sorológica à vacina contra hepatite $B$ nos pacientes candidatos ao transplante hepático, no Hospital Santa Isabel de Blumenau/SC. Métodos: Trata-se de um estudo realizado em duas etapas, no período entre março de 2011 e julho de 2012, no Hospital Santa Isabel. A primeira etapa trata-se de um estudo transversal observacional baseado na revisão de prontuários. A população foi composta por todos os pacientes candidatos ao transplante hepático que permaneciam listados até o dia 16 de março de 2011. As variáveis clínicas estudadas foram: idade, sexo, procedência, presença de anti-HBs, presença de anti-HBc, presença de $\mathrm{HBsAg}$ reagentes, escore MELD. A segunda etapa, trata-se de um estudo longitudinal composta por pacientes que se apresentaram suscetíveis na primeira etapa do estudo. As variáveis estudadas foram: realização de vacinação contra Hepatite $\mathrm{B}$, número de doses da vacina contra hepatite $B$, presença de anti-HBs pós-vacinal, classificação Child-Turcotte-Pugh e escore MELD. Resultados: Foram analisados 150 prontuários de todos os pacientes candidatos ao transplante hepático. A média de idade foi de 53,2 anos, e $64 \%$ da população estudada eram do sexo masculino. A prevalência do antiHBs reagente foi de $14,64 \%$, não mostrando qualquer relação significativa com o escore MELD e classificação Child-Turcotte-Pugh. Em relação à soroconversão, 21,4\% dos pacientes soroconverteram após, pelo menos, uma dose da vacina contra hepatite B. Conclusão: Houve baixa prevalência de anti-HBs nos candidatos ao transplante hepático. A literatura mostra que a soroconversão vacinal é superior à encontrada no estudo; no entanto, baseiase em pesquisas com a população geral. A vacinação nos pacientes candidatos ao transplante hepático deve ser preconizada, pois a soroconversão nesses pacientes garante proteção contra infecção pelo vírus da hepatite $B$. Há necessidade de maiores estudos para comparação de prevalência do Anti-HBs e soroconversão pós-vacinal desse marcador em pacientes candidatos ao transplante hepático.

Keywords: Hepatite B; Transplante Hepático; Anticorpos Anti-Hepatite B; Vacinas contra Hepatite B.

Instituição:

Universidade Regional de Blumenau, Blumenau/SC - Brasil

\section{Correspondência:}

Luiza Dadan Perini

Rua Bahia, 2140, Bairro Salto, Blumenau/SC/Brasil

Tel: (47) 9907-3260

E-mail: luiza_dadan@hotmail.com

Recebido em: 09/04/2013

Aceito em: 30/01/3014

\section{INTRODUÇÃO}

O primeiro transplante hepático em humanos foi realizado em 1963, nos Estados Unidos. ${ }^{1}$ Em 2002, foi realizado o primeiro transplante hepático em Santa Catarina, no Hospital Santa Isabel de Blumenau, que hoje é referência nacional nesse setor. ${ }^{2,3}$ No ano de 2011, Santa Catarina realizou $7,17 \%$ dos transplantes hepáticos realizados no Brasil. Esse número correspondeu à realização de 17,1 transplantes por milhão de população do estado de Santa Catarina, estando somente abaixo do estado do Ceará (18,6 transplantes por milhão de população). ${ }^{4}$ 
Prevalência do Anti-HBS e avaliação da resposta sorológica à vacina contra Hepatite B em pacientes candidatos ao transplante hepático no Hospital Santa Isabel de Blumenau/Santa Catarina

Desde a introdução do transplante hepático como alternativa terapêutica para tratamento da doença hepática em fase terminal, a escassez de doadores tornou-se questão importante devido à discrepância entre demanda e oferta de órgãos. ${ }^{5,6}$ Dados da United Network for Organ Sharing (UNOS) demonstram que 6.650 transplantes de fígado foram realizados no ano de 2006 e, ao mesmo tempo, 17.221 pacientes foram alocados na lista de espera para transplante hepático. Essa disparidade levou ao desenvolvimento de um sistema para priorizar o grande número de potenciais beneficiários em relação ao número disponível de doadores. ${ }^{7}$ Além do desenvolvimento de critérios para a inclusão dos pacientes na fila de transplante hepático, muitos serviços têm considerado a utilização de órgãos marginais, incluindo o uso de enxerto hepático Anti-HBc reagente e HbsAg não reagente como estratégia para aumentar o número de doadores. ${ }^{5,8}$

A infecção pelo vírus da hepatite $B$ (VHB) é uma das infecções mais comuns do gênero humano. Afeta um número estimado de 350 milhões de pessoas no mundo e é considerada uma das dez principais causas de morte. ${ }^{9,10}$ No Brasil, a soroprevalência do VHB é de 7,9\%. ${ }^{11}$ No padrão de distribuição da hepatite $B$ apresentado na região Sul, a região oeste de Santa Catarina apresenta prevalência moderada a alta, sendo maior que $7 \% .^{12}$

Nas áreas em que a prevalência de infecção pelo vírus da hepatite $B$ é alta, a utilização de órgãos de doadores Anti-HBc reagente e HBsAg não reagente pode ser uma solução para aumentar o número possível de doadores. Nesses casos, taxas variáveis de infecção pelo VHB de novo têm sido relatadas, dependendo do perfil sorológico do destinatário e do tipo de terapia preventiva empregada. A proteção contra infecção pelo VHB de novo foi associada à presença de anticorpos anti-HBc (indicativo de infecção prévia pelo VHB) e / ou Anti-HBs (indicativo de vacinação prévia ou infecção prévia pelo VHB). ${ }^{5}$

$\mathrm{O}$ anti-HBs reagente representa imunidade contra o VHB; sendo assim, é considerado fator protetor para pacientes candidatos ao transplante hepático. ${ }^{13}$ Segundo AvelinoSilva et al., enxertos de doadores Anti-HBc reagente e HBsAg não reagente são aparentemente seguros para o transplante em receptores vacinados (anti-HBs reagente), quando associados à terapia preventiva. ${ }^{5,14-16}$

A presença de anti-HBs reagente, além de ampliar a possibilidade de doadores para os candidatos ao transplante hepático, age na proteção contra infecção futura pelo VHB, já que os pacientes receberão órgãos e transfusão sanguínea potencialmente contaminados. Ao evitar infecção pelo vírus, há diminuição da incidência de hepatite crônica, cirrose, carcinoma hepatocelular e hepatite colestática fibrosante, impedindo a perda precoce do órgão transplantado. ${ }^{17}$
Os títulos de anti-HBs considerados protetores são iguais ou superiores a $10 \mathrm{mUl} / \mathrm{ml}$. Após as três doses da vacina, mais de $90 \%$ dos adultos jovens e mais de $95 \%$ das crianças e adolescentes desenvolvem respostas adequadas de anticorpos. ${ }^{18,19}$

Os objetivos deste estudo foram identificar a prevalência do anticorpo anti-HBs e avaliar a resposta sorológica à vacina contra hepatite $B$ nos pacientes candidatos ao transplante hepático, no Hospital Santa Isabel de Blumenau/SC.

\section{MATERIAIS E MÉTODOS}

Trata-se de estudo realizado em duas etapas, no período entre março de 2011 e julho de 2012, no Hospital Santa Isabel da cidade de Blumenau/SC.

\section{Primeira etapa do estudo}

A primeira etapa trata-se de um estudo transversal observacional, realizado entre março e novembro de 2011.

A população foi composta por todos os pacientes candidatos ao transplante hepático que permaneciam em lista até o dia 16 de março de 2011.

A coleta de dados foi realizada com base na revisão de prontuários. As variáveis clínicas estudadas foram: idade, sexo, procedência, presença de anti-HBs, presença de anti-HBc, presença de HBsAg reagentes e escore MELD (avaliado pelo valor $<18$ ou $\geq 18$, pois estudos afirmam que com pontuação acima de 18 , o paciente possui hepatopatia grave). ${ }^{20} \mathrm{~A}$ variável idade foi avaliada conforme a média e foi estratificada em intervalos de 10 anos, a partir de 20 anos.

O objetivo dessa primeira etapa foi identificar a prevalência do anti-HBs reagente nos pacientes candidatos ao transplante hepático.

Os resultados dos exames anti-HBs e anti-HBc foram obtidos através do teste ELISA realizados por laboratórios que seguem as exigências do Ministério da Saúde, do artigo 81, da Portaria 2600 de outubro de 2009. Os resultados dos marcadores sorológicos foram classificados como reagente ou não reagente. Considerou-se anti-HBs reagente quando a concentração de anti-HBs foi maior ou igual a $10,0 \mathrm{mUI} / \mathrm{ml}$.

Os perfis imunológicos foram classificados como: Sem vacina e sem exposição à hepatite $B$ (anti-HBc, antiHBs e HBsAg não reagentes), Curado da hepatite $B$ (anti-HBc e anti-HBs reagentes e HBsAg não reagente), Vacinado contra hepatite $B$ (anti-HBc e HBsAg não 
reagentes e anti-HBs reagente), Hepatite $B$ atual (anti$\mathrm{HBc}$ reagente ou não reagente, $\mathrm{HBsAg}$ reagente e anti$\mathrm{HBs}$ não reagente), Anti-HBc reagente isolado (anti-HBc reagente e HBsAg e anti-HBs não reagentes).

Os dados foram organizados em tabelas descritivas, contendo: frequências absolutas, frequências relativas, percentuais (proporções), médias, desvio padrão, mediana e intervalos de confiança.

As medidas foram estimadas em forma de intervalos de confiança para a proporção e para a média, com nível de confiança de 95\%. Para associar as variáveis, foi utilizado o teste Qui-quadrado de independência e aderência.

A significância estatística nos testes foi considerada com valor $P<0,05$. Para análise dos dados, foram utilizadas planilhas do Microsoft Excel versão 2007, contendo fórmulas desenvolvidas para cada situação e o software EPI INFO versão 3.5.3.

\section{Segunda etapa do estudo}

A segunda etapa trata-se de um estudo longitudinal, realizado entre novembro de 2011 e julho de 2012 .

A população foi composta por pacientes que apresentaram anti-HBs, anti-HBc e HBsAg não reagentes na primeira etapa do estudo. As variáveis estudadas foram: realização de vacinação contra Hepatite $B$, número de doses da vacina contra hepatite $B$, presença de anti-HBs pós vacinal, escore MELD e Child-Turcotte-Pugh.

O objetivo da segunda etapa foi verificar a soroconversão do anti-HBs após, pelo menos, uma dose da vacina contra Hepatite B. A classificação Child-Turcotte-Pugh (avaliada pela pontuação de 5-6, 7-9 e 10-15, correspondendo à classificação $\mathrm{A}, \mathrm{B}$ e $\mathrm{C}$, respectivamente) ${ }^{21}$ e escore MELD foram avaliados na amostra dos pacientes vacinados com, pelo menos, uma dose.

Os pacientes foram orientados a realizar a vacinação contra hepatite B até o mês de abril de 2012, seguindo protocolo do Centro de Transplante do Hospital Santa Isabel. Nos pacientes que realizaram no mínimo uma dose de vacinação foi pesquisado o anti-HBs no soro dos mesmos após um mês da última dose, ou seja, em maio de 2012. Todos os pacientes vacinados foram analisados quanto à presença do anti-HBs.

Em relação ao número realizado de doses da vacina, os pacientes foram classificados em: primeira dose, segunda dose e terceira dose realizadas.

$O$ resultado dos exames anti-HBs pós-vacinal foi obtido através do teste ELISA, realizado por laboratórios que seguem as exigências do Ministério da Saúde, do artigo 81, da Portaria 2600 de outubro de 2009. Foi considerado imunizado o indivíduo que apresentou no exame sorológico concentração de anti-HBs maior ou igual a $10,0 \mathrm{mUl} / \mathrm{ml}$.

Quanto ao anti-HBs pós-vacinal, os pacientes foram classificados em: Anti-HBs reagente e anti-HBs não reagente.

Foram considerados com critério de exclusão para essa etapa da pesquisa, os pacientes que evoluíram com óbito, os que não realizaram a vacina e os que não realizaram o exame de anti-HBs pós-vacinal.

Os indivíduos que participaram da segunda etapa da pesquisa receberam o Termo de Consentimento livre e esclarecido.

Os dados foram organizados em tabelas descritivas contendo: frequências absolutas, frequências relativas percentuais (proporções), médias, desvios padrão, mediana e intervalos de confiança.

As medidas foram estimadas em forma de intervalos de confiança para a proporção e para a média, com um nível de confiança de $95 \%$. Para associarem as variáveis, foi utilizado o teste Qui-quadrado de independência e de aderência.

A significância estatística nos testes foi considerada com valor $P<0,05$. Para análise dos dados foram utilizadas planilhas do Microsoft Excel versão 2007 contendo fórmulas desenvolvidas para cada situação e o software EPI INFO versão 3.5.3.

\section{RESULTADOS}

\section{Primeira etapa do estudo}

Foram analisados 150 prontuários de todos os pacientes candidatos ao transplante hepático que permaneciam em lista até o dia 16 de março de 2011, no Hospital Santa Isabel, de Blumenau.

A média de idade foi de 53 anos e dois meses, com desvio padrão de 9,56 para mais ou para menos. $O$ sexo masculino correspondeu a $64 \%$ da população estudada, sendo que a média de idade nesse grupo foi de 53 anos e quatro meses, com desvio padrão de aproximadamente 8,6 . Já no grupo feminino, a média de idade foi de 51 anos e 10 meses, com desvio padrão de aproximadamente 11,03 .

Quanto à procedência, $15,33 \%$ dos pacientes eram de Blumenau e $84,67 \%$ procederam de outras cidades do estado de Santa Catarina. O perfil dos pacientes conforme sexo, idade e procedência está ilustrado na tabela 1. 
Prevalência do Anti-HBS e avaliação da resposta sorológica à vacina contra Hepatite B em pacientes candidatos ao transplante hepático no Hospital Santa Isabel de Blumenau/Santa Catarina

Tabela 1 - Sexo, distribuição da idade por faixa etária e procedência dos pacientes do estudo.

\begin{tabular}{|c|c|c|c|}
\hline & $\begin{array}{c}\mathrm{N}^{\circ} \mathrm{de} \\
\text { Pacientes }\end{array}$ & $\%$ & IC (95\%) \\
\hline \multicolumn{4}{|l|}{ Sexo } \\
\hline Masculino & 96 & 64 & $(56,32-71,68)$ \\
\hline Feminino & 54 & 36 & $(28,32-43,68)$ \\
\hline Total & 150 & 100,0 & $\mathrm{Qui}^{2}=11,76 ; \mathrm{gl}=1 ; \mathrm{P}<0,01$ \\
\hline \multicolumn{4}{|l|}{ Idade (anos) } \\
\hline$<20$ & - & - & - \\
\hline 20 a 29 & 5 & 3,33 & $(0,46-6,21)$ \\
\hline 30 a 39 & 5 & 3,33 & $(0,46-6,21)$ \\
\hline 40 a 49 & 35 & 23,33 & $(16,56-30,1)$ \\
\hline 50 a 59 & 72 & 48,00 & $(40-56)$ \\
\hline 60 a 69 & 31 & 20,67 & $(14,19-27,15)$ \\
\hline 70 ou mais & 2 & 1,33 & $(0-3,17)$ \\
\hline Total & 150 & 100,00 & $\mathrm{Qui}^{2}=196,45 ; \mathrm{gl}=6 ; \mathrm{P}<0,01$ \\
\hline \multicolumn{4}{|l|}{ Mesorregiões } \\
\hline Vale do Itajaí & 55 & 36,67 & $(28,95-44,38)$ \\
\hline Sul Catarinense & 32 & 21,33 & $(14,78-27,89)$ \\
\hline $\begin{array}{l}\text { Grande } \\
\text { Florianópolis }\end{array}$ & 29 & 19,33 & $(13,01-25,65)$ \\
\hline Oeste Catarinense & 18 & 12 & $(6,8-17,2)$ \\
\hline Serrana & 8 & 5,33 & $(1,74-8,93)$ \\
\hline Norte Catarinense & 8 & 5,33 & $(1,74-8,93)$ \\
\hline Total & 150 & 100,00 & $\mathrm{Qui}^{2}=63,68 ; \mathrm{gl}=5 ; \mathrm{P}<0,01$ \\
\hline
\end{tabular}

A taxa de pacientes com anti-HBs reagente foi de $14,67 \%(P<0,01)$. A relação entre anti-HBs reagente $e$ idade, sexo e procedência dos pacientes está ilustrada na tabela 2. Dos pacientes com Anti-HBs reagente, $45,5 \%$ apresentaram proteção conferida pela vacinação prévia contra hepatite B (HBsAg-/anti-HBc-/Anti-HBs+) e $54,5 \%$ por hepatite B prévia (HBsAg-/anti-HBc+/Anti$\mathrm{HBs}+$ ) como ilustrado na tabela 3 .

A frequência de anti-HBc reagente foi de $20 \%$ e a de HBsAg reagente foi de $4 \%$ do total de pacientes. A tabela 3 demonstra o perfil imunológico dos pacientes candidatos ao transplante hepático. A maior prevalência de pacientes com anti-HBs, anti-HBc e HBsAg não reagente $(P<0,01)$ foi de $72 \%$, seguido de $9,3 \%$ de pacientes com anti-HBc reagente isolado. Oito por cento dos pacientes apresentaram perfil compatível com vacinados contra hepatite $B$ e $6,7 \%$ apresentaram perfil compatível com cura. Dos pacientes vacinados, $66,7 \%$ eram do sexo masculino; $50 \%$ apresentavam-se na faixa etária entre 50 e 59 anos, $25 \%$ na faixa etária entre 40 e 49 anos e $8,33 \%$ para cada uma das faixas etárias de 20 a 29 anos, de 60 a 69 anos e acima de 70 anos. Hepatite $\mathrm{B}$ atual apresentou prevalência de $4 \%$.
Tabela 2 - Relação entre o anti-HBs reagente e idade, sexo e procedência dos pacientes

\begin{tabular}{lccccc}
\hline & $\begin{array}{c}\text { Anti-HBS } \\
\text { Reagente } \\
(\mathbf{n = 2 2})\end{array}$ & $\mathbf{\%}$ & $\begin{array}{c}\text { Anti-HBS } \\
\text { Reagente } \\
(\mathbf{n = 1 2 8})\end{array}$ & $\mathbf{\%}$ & $\mathbf{p}$ \\
\hline Idade & & & & & \\
\hline$<20$ & - & - & - & - & - \\
20 a 29 & 1 & 4,5 & 4 & 3,1 & \\
30 a 39 & 0 & 0 & 5 & 3,9 & \\
40 a 49 & 4 & 18,2 & 31 & 24,2 & 0,58 \\
50 a 59 & 12 & 54,5 & 60 & 46,9 & \\
60 a 69 & 3 & 13,6 & 28 & 21,9 & \\
70 ou mais & 2 & 9,1 & 0 & 0 & \\
\hline Total & $\mathbf{2 2}$ & $\mathbf{1 0 0}$ & $\mathbf{1 2 8}$ & $\mathbf{1 0 0}$ & \\
\hline Sexo & & & & & \\
\hline Masculino & 18 & 81,8 & 78 & 60,9 & 0,05 \\
Feminino & 4 & 18,2 & 50 & 39,1 & \\
\hline Total & $\mathbf{2 2}$ & $\mathbf{1 0 0}$ & $\mathbf{1 2 8}$ & $\mathbf{1 0 0}$ & \\
\hline Mesorregióes & & & & & \\
\hline Serrana & 1 & 4,5 & 7 & 5,5 & \\
Vale do Itajaí & 4 & 18,2 & 51 & 39,9 & \\
Sul Catarinense & 3 & 13,6 & 29 & 22,6 & \\
Norte Catarinense & 1 & 4,5 & 7 & 5,5 & 0,03 \\
Oeste Catarinense & 3 & 13,6 & 15 & 11,7 & \\
Grande Florianópolis & 10 & 45,5 & 19 & 14,8 & \\
\hline Total & $\mathbf{2 2}$ & $\mathbf{1 0 0}$ & $\mathbf{1 2 8}$ & $\mathbf{1 0 0}$ & \\
\hline & & & & & \\
\hline
\end{tabular}

Tabela 3 - Distribuição dos pacientes segundo perfil imunológico (Anti-HBs e Anti-HBc e HBsAg)

\begin{tabular}{|l|l|c|c|}
\hline $\begin{array}{c}\text { Anti-HBs/Anti-HBc/ } \\
\text { HBsAg }\end{array}$ & \multicolumn{1}{|c|}{ Perfil Imunológico } & $\begin{array}{c}\text { No de } \\
\text { Pacientes }\end{array}$ & $\mathbf{\%}$ \\
\hline$(+) /(+) /(-)$ & Curado de hepatite B & 10 & 6,7 \\
$(+) /(-) /(-)$ & Vacinado para hepatite B & 12 & 8,0 \\
$(-) /(+/-) /(+)$ & Hepatite B atual & 6 & 4,0 \\
$(-) /(+) /(-)$ & Anti-HBc reagente isolado & 14 & 9,3 \\
$(-) /(-) /(-)$ & Anti-HBs e Anti-HBc e & 108 & 72,0 \\
\hline Total & HBsAg não reagentes & $\mathbf{1 5 0}$ & $\mathbf{1 0 0}$ \\
\hline
\end{tabular}

Tabela 4 - Anti-HBs pós vacinação contra hepatite B relacionado ao número de doses recebidas da vacina contra hepatite $B$

\begin{tabular}{|l|c|c|c|c|}
\hline \multirow{2}{*}{$\begin{array}{l}\text { Anti-HbS pós- } \\
\text { vacinação contra } \\
\text { hepatite B }\end{array}$} & \multicolumn{4}{|c|}{ Número de doses da vacina conra hepatite B } \\
\hline Reagente & $0(0,0 \%)$ & $1(16,7 \%)$ & $5(25,0 \%)$ & $6(21,4 \%)$ \\
\hline Não-reagente & $2(100,0 \%)$ & $5(83,3 \%)$ & $15(75,0 \%)$ & $22(78,6 \%)$ \\
\hline Total & $2(100,0 \%)$ & $6(100,0 \%)$ & $20(100,0 \%)$ & $28(100,0 \%)$ \\
\hline
\end{tabular}


Em relação ao MELD pré-vacinal, não foram encontrados resultados estatisticamente significativos $(P>0,05)$. A frequência de reatividade do anti-HBs foi equiparável nos dois subgrupos estudados, com um resultado de aproximadamente $15 \%$, como aventado na tabela 5 .

Tabela 5 - Relação entre MELD, Child e o Anti-HBs

\begin{tabular}{lccccc}
\hline & $\begin{array}{c}\text { Anti-HBs } \\
\text { Reagente }\end{array}$ & $\begin{array}{c}\text { Anti-HBs } \\
\text { Não-reagente }\end{array}$ & Perda & Total & P \\
\hline Pré-vacinal & & & & \\
\hline MELD & & & & \\
$<18$ & $20(90,9 \%)$ & $115(89,8 \%)$ & - & $135(90 \%)$ & 0,1958 \\
$\geq 18$ & $2(9,1 \%)$ & $12(9,4 \%)$ & - & $14(9,3 \%)$ & \\
Não informado & - & $1(0,8 \%)$ & - & $1(0,7 \%)$ & \\
Pós-vacinal & & & & & \\
\hline MELD & & & & & \\
$<18$ & $6(100 \%)$ & $20(90,9 \%)$ & $34(97,1 \%)$ & $60(95,2 \%)$ & 0,4750 \\
$\geq 18$ & - & $2(9,1 \%)$ & $1(2,9 \%)$ & $3(4,8 \%)$ & \\
Child & & & & & \\
Classe A & $3(50 \%)$ & $3(13,6 \%)$ & $3(8,6 \%)$ & $9(14,3 \%)$ & 0,3964 \\
Classe B & $3(50 \%)$ & $2(9,1 \%)$ & $7(20 \%)$ & $12(19 \%)$ & \\
Classe C & - & $3(13,6 \%)$ & $4(11,4 \%)$ & $7(11,1 \%)$ & \\
Não informado & - & $14(63,6 \%)$ & $21(60 \%)$ & $35(55,6 \%)$ & \\
\hline
\end{tabular}

\section{Segunda etapa do estudo}

$\mathrm{Na}$ segunda etapa foram incluídos pacientes com todas as sorologias não reagentes (HBsAg-/AntiHBc-/ AntiHBs-), totalizando 108 pacientes. A análise do número de doses da vacina realizadas após orientação apresentou os seguintes resultados: dos 108 pacientes com todas as sorologias não reagentes, 63 (58,3\%) pacientes realizaram pelo menos uma dose da vacina contra Hepatite B, $45(41,7 \%)$ foram excluídos do estudo pelos seguintes motivos: recusa na realização da vacinação, sem informação e óbito. Dos pacientes que realizaram pelo menos uma dose da vacina, a frequência de pacientes que realizaram a primeira, segunda e terceira dose foi, respectivamente, $7,9 \%$, $23,8 \%$ e $68,3 \%$.

Em relação ao anti-HBs pós-vacinal, os resultados encontrados foram: $21,4 \%$ dos pacientes apresentaram anti-HBs reagente; $78,6 \%$ dos pacientes apresentaram anti-HBs não reagente; 35 pacientes foram excluídos do estudo, devido a não realização de exame do anti-HBs pós-vacinal por não comparecimento. Esses resultados estão ilustrados na tabela 4 . O algoritmo dos resultados do estudo está ilustrado na figura 1.

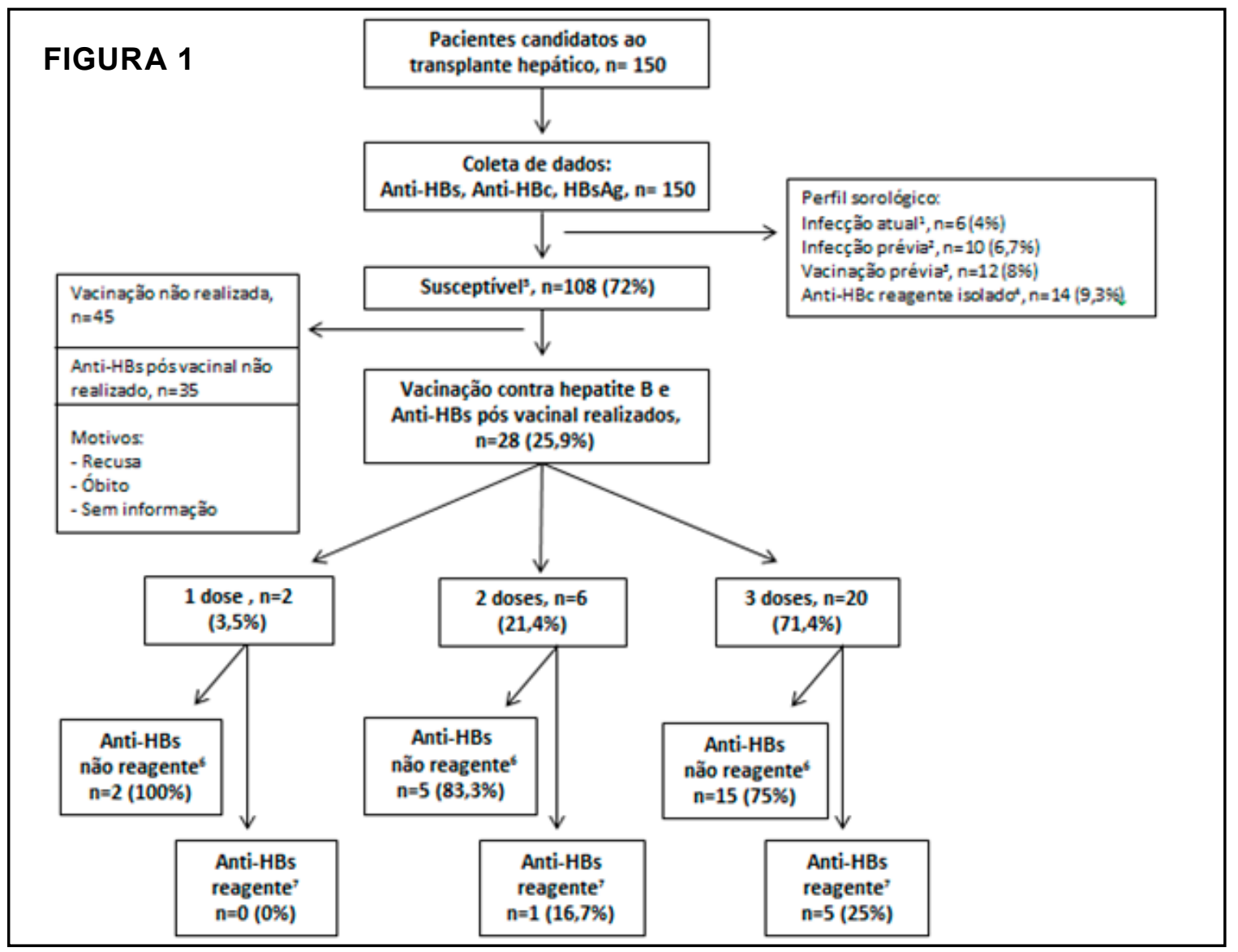


Prevalência do Anti-HBS e avaliação da resposta sorológica à vacina contra Hepatite B em pacientes candidatos ao transplante hepático no Hospital Santa Isabel de Blumenau/Santa Catarina

Dos pacientes com MELD pós-vacinal, embora com valor estatisticamente não significativo maior ou igual a 18 , nenhum apresentou positividade no anti-HBs, enquanto no outro subgrupo, $10 \%$ tornaram-se imunizados. No atual estudo, o parâmetro Child também não demonstrou relevância estatística; porém, do subgrupo Child $\mathrm{C}$, nenhum adquiriu o anti-HBs, conforme segue ilustrado na tabela 5. Pacientes classificados como Child A mostraram uma resposta de $33,3 \%$ à vacinação, enquanto que Child $B, 25 \%$ obtiveram imunidade contra o vírus.

\section{DISCUSSÃO}

A maioria dos pacientes do estudo, candidatos ao transplante hepático, encontrava-se no intervalo entre 50 e 59 anos, sendo a média de idade 53 anos e dois meses. Esse perfil pode ser justificado pelo fato da grande parte dos pacientes estudados ser portadora de doenças crônicas. Quanto ao sexo, 64\% dos pacientes eram do sexo masculino.

O anti-HBs é o anticorpo associado à cura e ao desenvolvimento de imunidade contra o VHB. Quando presente isoladamente, indica desenvolvimento da imunidade vacinal ao $\mathrm{VHB} . .^{18} \mathrm{O}$ anti-HBs reagente pode ser considerado fator protetor contra a hepatite B. Esse fato demonstra a importância desse marcador reagente nos pacientes que serão submetidos ao transplante hepático, pois estes receberão transfusão sanguínea e um órgão que, apesar de testes realizados, podem conter o VHB em janela imunológica, ou seja, HBsAg não reagente. Além disso, segundo Avelino-Silva et al., ${ }^{5}$ enxertos de doadores Anti-HBc reagente e HBsAg não reagente são aparentemente seguros para transplante em receptores vacinados, quando associados à terapia preventiva. Dessa forma, pacientes com Anti-HBs reagente possuem maior disponibilidade para órgãos doadores.

A prevalência de anti-HBs reagente em pacientes candidatos ao transplante hepático desse estudo foi $14,67 \%$ dos pacientes pesquisados. Não foram encontrados estudos de prevalência do anti-HBs em pacientes candidatos ao transplante hepático na literatura pesquisada, o que demonstra a importância da realização de outros estudos semelhantes a esse, para comparação de dados e avaliação da proteção dos candidatos ao transplante hepático. Porém, a baixa prevalência da soroconversão encontrada pode ser explicada pela alta frequência de pacientes cirróticos, os quais respondem menos favoravelmente à vacinação contra hepatite $\mathrm{B}$, apresentando taxa de soroconversão em aproximadamente $38,5 \%$ dos pacientes. ${ }^{22}$
A prevalência do anti-HBs reagente foi maior na faixa etária entre 50 e 59 anos $(54,5 \%)$, no sexo masculino $(81,8 \%)$ e nos indivíduos provenientes da Grande Florianópolis (45,5\%). Sendo a região oeste de Santa Catarina considerada de alta endemicidade para hepatite B (superior a $7 \%$ ), esperava-se que a maior prevalência do anti-HBs fosse encontrada nessa região. ${ }^{23}$ Essa discordância deve-se ao fato de que a maioria dos pacientes do estudo não era procedente dessa região, e que muitos pacientes mudam-se para o Vale do Itajaí pela maior facilidade de acompanhamento médico.

Em relação aos pacientes com anti-HBs não reagente, aqueles acima de 40 anos representaram a maioria $(79,4 \%)$. Desse modo, a baixa prevalência de anticorpo anti-HBs na população em estudo pode ser justificada pela não realização da vacinação contra hepatite $B$, já que a vacina passou a ser oferecida pelo Sistema Único de Saúde (SUS), a partir de agosto de 1989. ${ }^{24,25}$ Atualmente, preconiza-se a vacinação universal na infância, iniciada logo após o nascimento e em crianças e adolescentes de um a 19 anos. Deve-se realizar também a vacinação em grupos de pacientes específicos, sendo os candidatos ao transplante hepático um deles. ${ }^{18}$

Outro fator que poderia justificar a prevalência do AntiHBs nos pacientes do estudo é a duração da imunidade da vacina contra hepatite B. Segundo a literatura, a queda dos títulos de anticorpos é mais intensa no primeiro ano após a vacinação; entretanto, espera-se que $30 \%$ a $60 \%$ dos vacinados que soroconverteram tenham redução dos títulos de anti-HBs abaixo de $10 \mathrm{mUl} / \mathrm{ml}$ em cinco anos após a vacinação. Nesse caso, os pacientes do estudo podem ter realizado o esquema vacinal prévio, porém, como se trata de pacientes adultos e idosos, com o passar dos anos houve queda dos títulos de anticorpos e consequente negativação da sorologia. ${ }^{18}$

Em relação ao anti-HBc, 9,3\% dos pacientes apresentaram o marcador anti-HBc reagente isolado, o que significa um perfil sorológico indefinido. Esse perfil pode indicar infecção antiga, em que o anti-HBs já não é mais detectado, portadores crônicos com baixa produção de HBsAg (hepatite B oculta) ou reação cruzada com anticorpos produzidos pela infecção pelo vírus da hepatite $C .{ }^{18,26}$

Do total de pacientes estudados, $72 \%$ apresentaram-se suscetíveis (anti-HBs, anti-HBc, HBsAg não reagentes), o que significa que o paciente não foi vacinado, não foi exposto ao VHB, encontra-se em janela imunológica ou não fez soroconversão após vacinação. Esses pacientes foram orientados a realizar três doses da vacina contra hepatite $B$, preferencialmente antes do procedimento para possível soroconversão e proteção contra futura exposição ao VHB. ${ }^{27}$ Todos eles realizaram pelo menos 
Marcelo Augusto Scheidemantel Nogara, Luiza Dadan Perini, Fabiane Miura Ogg De Salles, Maria Gabriela Ortiz de Noronha, Guilherme Bortolli Seter, Camila Ribeiro Batista

uma dose da vacina contra a hepatite B; entretanto, somente 28 desses realizaram o exame do anti-HBs pós-vacinal.

Os motivos da não realização do anti-HBs pós-vacinal foram: pacientes que evoluíram a óbito durante o estudo; pacientes que não transmitiram a informação e pacientes que se recusaram a realizar o exame.

Segundo a literatura, três doses da vacina contra hepatite $B$ induzem títulos protetores de anticorpos (anti-HBs maior ou igual a $10 \mathrm{mUl} / \mathrm{ml}$ ) em mais de $90 \%$ dos adultos e jovens sadios, e em mais de $95 \%$ dos lactentes, das crianças e dos adolescentes. ${ }^{18}$ No estudo de Ferraz et al., com pacientes sem cirrose, $43 \%$ dos participantes apresentaram soroconversão após a primeira dose vacinal. ${ }^{28}$ No Uruguai, Arca et al. observaram que $93,4 \%$ dos participantes com esquema vacinal completo tinham concentrações de anti-HBs > que $1000 \mathrm{mUl} / \mathrm{ml}^{29}$

A frequência da soroconversão no presente estudo foi proporcional ao maior número de doses realizadas da vacina; no entanto, foi menor quando comparada à população geral. A frequência de soroconversão com uma, duas e três doses vacinais foi de 0\%, 16,6\% e $25 \%$, respectivamente. Apesar da baixa frequência de soroconversão do anti-HBs e o alto preço da vacinação, esta é altamente efetiva na prevenção de doenças hepáticas crônicas e agudas nos imunizados. ${ }^{30}$

A baixa soroconversão dos pacientes do estudo pode ser explicada pela idade e pela presença de doença crônica nessa população. A soroconversão ocorre em proporção inversa à idade, de modo que níveis protetores de anticorpos são encontrados em apenas $70 \%$ das pessoas entre 50 e 59 anos e em $50 \%$ daqueles com mais de 60 anos. Esse fato deve-se à senescência do sistema imune. ${ }^{18,31}$ A soroconversão também diminui com a presença de insuficiência renal, diabetes, doença hepática crônica, infecção pelo vírus da imunodeficiência humana, tabagismo e obesidade..$^{18}$

De acordo com Roni et al., dos pacientes com doença hepática crônica estudados, somente $60 \%$ responderam à vacinação, enquanto que $19 \%$ responderam pobremente e $21 \%$ não obtiveram resposta alguma. Pacientes com doença hepática crônica devido ao álcool demonstraram pior resposta (44\%) quando comparados ao quadro de etiologia criptogênica (69\%). A severidade daquela doença também é um preditor.

Dos pacientes classificados como Child classe A, $88 \%$ responderam bem à vacinação, enquanto que dos pacientes com Child classe B, somente 33,3\% obtiveram a mesma resposta. ${ }^{32}$ No estudo em questão, observou-se frequência menor da resposta à vacinação nos subgrupos quando comparado à literatura. $\mathrm{O}$ resultado do subgrupo Child $A$ equiparou-se ao Child $B$ do estudo supracitado.

Esse quadro deve-se, provavelmente, à grande perda de pacientes durante o estudo. Outro indicador de severidade da hepatopatia é o MELD. ${ }^{20}$ No estudo, foi encontrada alguma resposta à vacina no subgrupo MELD <18, enquanto que no outro, não houve resposta.

O grande número de perdas durante a realização da pesquisa prejudicou o resultado e análise final do estudo, pois muitos pacientes não realizaram a vacinação ou não compareceram ao exame. Outro viés do estudo foi a impossibilidade de realização do anti-HBs pós-vacinal após todos os pacientes terem recebido o esquema completo de vacinação contra hepatite $B$. Esse viés, no entanto, não pode ser evitado pelo curto tempo hábil para realização deste trabalho e pelo fato dos pacientes realizarem o transplante hepático antes da finalização da pesquisa.

Segundo a literatura, entre aqueles que não obtiveram resposta vacinal adequada após as três doses iniciais, $25 \%$ a $40 \%$ respondem após uma única dose adicional e $50 \%$ a $70 \%$ respondem ao esquema de três doses. Esse perfil de resposta não deve ser equivalente ao da população de estudo, já que a população consiste em candidatos ao transplante hepático. ${ }^{18}$

\section{CONCLUSÃO}

Os resultados da pesquisa demonstraram baixa prevalência de anti-HBs nos candidatos ao transplante hepático. Não foi possível realizar a comparação dos resultados com outros trabalhos, devido à inexistência de pesquisas sobre prevalência desse marcador em pacientes candidatos ao transplante hepático.

A literatura mostra que a soroconversão vacinal é superior à encontrada no estudo; no entanto, baseia-se em pesquisas com a população geral. Esse achado é importante, pois demonstra que a vacinação preconizada na infância é mais eficaz tanto pela maior resposta imunológica quanto pela ausência de doença crônica.

Dos 20 pacientes com anti-HBs não reagente no início do estudo e que realizaram corretamente as três doses da vacina contra hepatite B, $25 \%$ soroconverteram. Dessa forma, pode-se concluir que um quarto dos pacientes que realizaram as três doses da vacina contra hepatite B apresentaram anti-HBs reagente no final do estudo.

Há necessidade de maiores estudos para comparação de prevalência de Anti-HBs e soroconversão pósvacinal desse marcador em pacientes candidatos ao transplante hepático. 
Prevalência do Anti-HBS e avaliação da resposta sorológica à vacina contra Hepatite B em pacientes candidatos ao transplante hepático no Hospital Santa Isabel de Blumenau/Santa Catarina

\section{ABSTRACT}

Purpose: Anti-HBs positive is a protective factor for patients eligible for liver transplantation, as it poses immunity against the hepatitis B virus (HBV). After three doses of the vaccine more than $90 \%$ of young adults develop adequate Anti-HBs antibody responses. The aims of this study were to identify the prevalence of anti-HBs and assess the antibody response to hepatitis $B$ vaccine in patients eligible for liver transplantation at Hospital Santa Isabel of Blumenau/ SC. Methods: This study was conducted in two steps during the period between March 2011 and July 2012, at Hospital Santa Isabel in Blumenau / SC. The first step was an observational cross-sectional study based on medical chart review. The population consisted of all patients eligible for liver transplantation who remained on the list until March 16, 2011. The clinical variables studied were: age, gender, origin, presence of anti-HBs, presence of anti-HBc and presence of HBsAg, MELD score. The second step was a longitudinal study, composed by patients who were susceptible in the first stage of the study. The variables studied were: presence of Hepatitis B vaccination, amount of doses of the hepatitis B vaccine, presence of after vaccination anti-HBs positive, Child-Turcotte-Pugh classification and MELD score. Results: We reviewed 150 medical charts of all patients eligible for liver transplantation. The mean age was 53.2 years, and $64 \%$ of the studied population were male. The prevalence of anti-HBs positive was $14.64 \%$, showing no significant relationship both with the MELD score and the Child-Turcotte-Pugh classification. Regarding the seroconversion, $21.4 \%$ of patients seroconverted after at least one dose of the vaccine against hepatitis $\mathrm{B}$. Conclusion: There was low prevalence of anti-HBs positive in patients eligible for liver transplantation. The literature shows that the vaccine seroconversion is higher than data found in this study; however, it is based on surveys of the general population. Vaccination in patients eligible for liver transplantation should be recommended, as the seroconversion of those patients provides protection against infection by hepatitis $B$. Further studies are required to compare the prevalence of anti-HBs seroconversion after vaccination and such marker in patients eligible for liver transplantation.

Keywords: Hepatitis B; Liver Transplantation; Hepatitis B Antibodies; Hepatitis B Vaccines.

\section{REFERÊNCIAS}

1. Mies S. Transplante de fígado. Rev Ass Med Brasil. 1998; 44:127-34.

2. Galvão J. O segmento de saúde para o desenvolvimento regional no município de Blumenau-SC: a participação do Hospital Santa Isabel [dissertação]. Blumenau: Universidade Regional de Blumenau; 2003.

3. Nogara MAS, Wiederkher J, Benghi RAC, Zalli M, Helena ETS. Avaliação dos transplantados hepáticos no estado de Santa Catarina no período de agosto de 2002 a janeiro de 2008. J Bras Transp. 2009; 12:1144-8.

4. Garcia VD (Ed.). Transplantes de Fígado por Estado. Registro brasileiro de Transplante. 2011;17:11.

5. Avelino-Silva VI, D’Albuquerque LAC, Bonazzi PR, Song ATW, Miraglia JL, de Brito Neves A, et al. Liver transplant from Anti$\mathrm{HBc}$-positive, HBsAg-negative donor into HBsAg-negative recipient: is it safe? A systematic review of the literature. Clin Transplant, 2010;24:735-46.

6. Lopez-Navidad A, Caballero F. Extended criteria for organ acceptance. Strategies for achieving organ safety and for increasing organ pool. Clin Transplant. 2003;17:308-24.

7. Mullhaupt B, Dimitroulis D, Gerlach JT, Clavien P. Hot topics in liver transplantation: Organ allocation - extended criteria donor - living donor liver transplantation. Journal of Hepatology. 2008;48:58-67.
8. Hospital Israelita Albert Einsten. Avaliação e cuidados no transplante hepático. 2009;3-38.

9. Cooreman MP, Leroux-Roels G, Paulij WP. Vaccine and Hepatitis $\mathrm{B}$ immune globulin-induced escape mutations of hepatitis B virus surface antigen. J Biomed Sci, Amsterdam. 2000;8:237-47.

10. Torbenson M, Thomas DL. Occult hepatitis B. Infectious diseases. 2002;2:479-86.

11. Tanaka J. Hepatitis B epidemiology in Latin America. Vaccine. 2000;18:17-9.

12. Barros Júnior GM. Hepatite crônica B oculta: estudo clínico, epidemiológico, histopatológico e molecular em doentes com diagnóstico de hepatite crônica da demanda da Fundação de Medicina Tropical do Amazonas [dissertação]. Manaus: Universidade do Estado do Amazonas; 2005.

13. Dhedin N, Douvin C, Kuentz M, Saint M, Marie F; Reman O, et al. Reverse seroconversion of hepatitis $\mathrm{B}$ after allogenic bone marrow transplantation: a retrospective study of 37 patients with pretransplant anti-HBs and anti-HBc. Tranplantation. 1998; 66:616-9.

14. Brock GN, Mostajabi F, Ferguson N, Carrubba CJ, Eng M, Buell JF, et al. Prophylaxis against de novo hepatitis B for liver transplantation utilizing hep $\mathrm{B}$ core $(+)$ donors: does hepatitis $\mathrm{B}$ immunoglobulin provide a survival advantage? European Society for Organ Transplantation. 2011;24:570-81. 
15. Burbach GJ, Bienzle U, Neuhaus R, Hopf U, Metzger WG, Pratschke J, et al. Intravenous or intramuscular anti-HBs immunoglobulin for the prevention of hepatitis B reinfection after orthotopic liver transplantation. Transplantation. 1997;63:478-80.

16. Saab S, Waterman B, Chi AC, Tong MJ. Comparison of Different Immunoprophylaxis Regimens After Liver transplantation with Hepatitis B Core Antibody-Positive Donors: A Systematic Review. Liver transplantation. 2010;16:300-7.

17. Papaiordanou F, Ribeiro-Junior MAF, Saad WA. Prevenção do carcinoma hepatocelular. ABCD, arq. bras. cir. dig., São Paulo. 2009;22:115-9.

18. Focaccia R. Tratado de hepatitis virais. São Paulo: Atheneu; 2007.

19. Scaramuzzi DR. Vacina contra hepatite B. Rev Assoc Med Bras. 2006;52:281-91.

20. Merion R. When Is a Patient Too Well and When Is a Patient Too Sick For a Liver Transplant? Liver Transpl. 2004;10:69-73.

21. Angermayr B, Cejna M, Karnel F, Gschwantler M, Koenig F, Pidlich J, et al. ChildPugh versus MELD score in predicting survival in patients undergoing transjugular intrahepatic portosystemic shunt. Gut. 2003;52:879-85

22. Koślińska-Berkan E, Kuydowicz J. The comparison of the humoral response among the patients with liver cirrhosis and steatosis of the liver after HBV vaccination. Przegl Epidemiol. 2006;60(2):199-203.

23. Garcia LP, Facchini LA. Vacinação contra a hepatite B entre trabalhadores da atenção básica à saúde. Cad. Saúde Pública. 2008;24:1130-40.

24. Diretoria De Vigilância Epidemiológica. Hepatite B: Informações para divulgação junto à imprensa Catarinense. Santa Catarina. 2012.
25. Kiesslich D, Fraiji NA, Crispim MA, Pereira FR, Martinho AC, Campello SC, et al. Prevalência de marcadores sorológicos e moleculares do vírus da hepatite $\mathrm{B}$ em gestantes do Estado do Amazonas, Brasil. Epidemiologia e Serviços de Saúde. 2003;12:155-64.

26. Wohlfahrt AB, Wohlfahrt AB, Beck ST, Foletto A, Ceccim A. Determinação do marcador Anti-HBc na prevenção da transmissão transfusional do vírus da Hepatite B: importancia e implicações. RBAC. 2010;42:269-72.

27. Souto FJD, Rodrigues EN, Fortes HM, Saldanha AA. Soroconversão do anti-HBs após vacina contra hepatite B em doadores de sangue $\mathrm{HBs}$ antígeno-negativos anti-HBc-positivos na rede pública de saúde, Mato Grosso, Brasil. Revista de Patologia Tropical. 2006;35:205-11.

28. Ferraz MLG, Silva AEB, Kemp VL, Cruz CN, Guimaräes RX. Avaliação da resposta imunológica à vacina contra hepatite $\mathrm{B}$ em profissionais da área da saúde. Rev. Assoc. Med. Bras. 1992;38:5-8.

29. Arca M, Gadea FA, Gabioud JC, Labalta CR, Oertlinger S, Sánchez LM. Tamizaje de marcadores para hepatitis $b$ pre y post vacunación en el hospital de C. de Uruguay, Argentina. Acta Bioquim Clin Lati-noam. 1998;32:377-82.

30. Weinbaum CM, Williams I, Mast EE, et al. Recommendations for Identification and Public Health Management of Persons with Chronic Hepatitis B Virus Infection. Morbidity and Mortality Weekly Report (MMWR). 2008;57:1-20.

31. Mast EM. Hepatitis B vaccine. In: Plotkin S, Orenstein. (Eds.). Vaccines. 4 ed. Philadelphia: WB SAUNDERS, 2004. p. 299337.

32. Roni DA, Pathapati RM, Kumar AS, et al. Safety and Efficacy of Hepatitis B Vaccination in Cirrhosis of Liver. Advances in Virology. 2013;2013:1-5.

\section{O trabalho foi aprovado pelo Comitê de Ética em dezembro de 2010, pelo protocolo 198-10.}

